\title{
A Master Key to Assess Stroke Consequences Across Species: The Adhesive Removal Test
}

\author{
Valentine Bouet and Thomas Freret \\ Université de Caen Basse-Normandie
}

France

\section{Introduction}

\subsection{History of the adhesive removal test}

Long-term consequences of stroke are often dramatically disabling for daily living but also costly for public health. Stroke research is therefore a priority for most industrialized countries. Therefore and paradoxically, the current situation is disappointing for the patients but stimulating for the researchers who have to figure out new therapies and assess their efficiency. The major initial problem to solve for clinicians when a stroke occurs is to rapidly distinguish between ischemia and hemorrhage to decide for the reperfusion of the brain or for the bleeding stop. Therefore, the first step of stroke treatment takes place within a very short-term and tiny time window (even if it was recently extended to 4 hours for thrombolysis). Next steps of the treatments are directed to avoid another stroke and to promote rehabilitation. For this latter, unfortunately, the treatments are scarce and although many different options have been tested (kinesiotherapy, speech therapy etc.), the situation is still unsatisfactory. News fields of research tried to find out new therapies aimed at improving neurogenesis, implanting stem cells or grafts, or stimulating trophic and growth factors, but in vain, transfer from animal to human is still disappointing. Another way to deal with treatment of stroke is to assess what happens in those patients who recover spontaneously. Indeed, around 33\% of stroke patients spontaneously recover after stroke but the mechanisms involved in the recovery, not elucidated till now, could be interesting to promote. To assess these mechanisms, it is of a major importance to be able to measure longterm functional deficits in animal models of stroke, which is by the way a strong recommendation from the STAIR Roundtables (STAIR, 1999; STAIRIII, 2001).

According to the brain structures infracted, stroke induces many different symptoms affecting physiological, sensori-motor, and/or cognitive functions. In experimental studies, it is possible to induce different models of stroke and to assess with many different ways their consequences. However, given the extraordinary spontaneous recovery displayed by animals, especially rodents, it is not always possible to highlight long-term deficits. Indeed, small brain lesions induce deficits that either cannot be detected by global behavioral tests or only for a short term period after stroke (limb placing test, neurological score). That is the reason why news tests have been designed to assess functional recovery in animals. Among the various behavioral tests developed so far, the adhesive removal test and all its variations (sticky-tape test, bilateral asymmetry test) have been proved to be one of the most efficient to highlight tiny long term sensori-motor deficits. Originally brought back from bedside to 
bench by Schallert et al. (Schallert et al., 1982), this test is derived from a neurologic exam routinely used by clinicians, namely the Double Simultaneous Stimulation (DSS). In fact, the DSS has been initially developed by clinicians to highlight the contralateral neglect syndrome which is observed in patients after damage to the parietal cortex (Heilman et al., 2000). During the DSS examination, the patient has to attend to and identify paired of sensory stimuli that are applied to both sides of the body at the same time. Stimuli can be visual, auditive or tactile. Extinction to double simultaneous tactile stimulation (tactile DSS) was first reported at the beginning of the 80's (Schallert et al., 1982). Tactile extinction is the failure to perceive and/or report tactile stimulation on the body side contralateral to a brain lesion when the homologous, ipsilesioned region is stimulated simultaneously; whereas no defect in tactile perception occurs with unilateral stimulation. Interestingly, tactile extinction showed to be promising as a predictor of post-stroke functional outcome (Rose et al., 1994). In the way of improving the functional assessment in experimental stroke models, adhesive removal test has been first adapted to rats about a quarter of century ago (Schallert et al., 1982). The stimulus employed was then tactile, consisting of adhesive tapes applied to different parts of the animal body (forelimbs, hindlimbs and snout). It measures sensory functions, sensory neglect and motor functions independently for the left and the right side. Initially designed to highlight deficit induced by unilateral nigrostriatal damage, its range of application has been extended to several brain disorders (Parkinson disease, brain trauma, spinal cord lesion), including stroke. Giving advantage to assess sensory and motor deficits, free from postural bias and circling behaviors, the adhesive removal test has been highly used in rats to a large extent. Interestingly, long-term after stroke, while many tests are not sensitive enough to measure any deficit because of an apparent full recovery, the adhesive removal test is one of the rare tests powerful enough to give an objective score of the functional deficit. Besides, the deficits observed after stroke very well mimics the extinction to tactile double simultaneous stimuli reported in stroke patients (Schallert et al., 1982). Owing to its success in rats, the adhesive removal test has been afterward developed in other animal species. Thus, in the beginning of the 90's, the test was adapted to a nonhuman primate species, the marmoset (Callithrix jacchus) (Annett et al., 1992; Marshall \&Ridley, 1996). Used then in gerbils (Ishibashi et al., 2003) and even dogs (Quaranta et al., 2004), it has been lastly developed in mice (Bouet et al., 2009; Bouet et al., 2007; Starkey et al., 2005). Thus, beyond its effectiveness to highlight long term, and consequently tiny, sensory and motor deficit, the adhesive removal test is adaptable to several animal models, which is a fundamental advantage to translate animal research to clinical application.

Convinced that this test could offer even more than it has already did, we will along this chapter give an overlook of the way it has to be performed and the results you may obtained in several animal models of stroke: mice, rats, and marmosets (Bouet et al., 2009; Bouet et al., 2010; Bouet et al., 2007; Freret et al., 2009; Freret et al., 2008; Freret et al., 2006; Freret et al., 2006), i.e. the most used species in neuroscience research.

\section{Achievement of adhesive removal test across laboratory species}

\subsection{Species}

As stated before, the adhesive removal test has been already developed in a wide range of animal species, from nonhuman primate to rodents (rats, mice, gerbils). This test can theoretically be performed in any species that is anatomically able to remove a piece of adhesive pasted on its body. However, according to the species concerned, technical details 
have to be adjusted, notably the size of adhesives tapes, but also the animal's body part that will be concerned by tactile stimulation depending on whether the species is purely a quadruped or not (see below).

\subsection{Training and age}

Although training sessions are not mandatory, they are highly recommended. Training decreases anxiety related to the test, and therefore decreases the probability that the animal will urinate and defecate during the subsequent test phase, allowing researchers to obtain an optimal level of performance. It is beside of prime importance to maintain consistency in the testing environment because small changes may impact on animals' emotional state and consequently on the functional outcome. Additionally, training allows indentifying any preoperative asymmetries that would induce a bias in the interpretation of the data. Finally, training also decreases inter-individual variability, making performances homogenous. Once the animals display good performances, only deficits due to the insult are compared, without learning effect. Furthermore, it is important that sham-operated and experimental animals are trained to the task, because surgery itself could induce a slight change in performances. Besides, doing so allow to use each animal performance as its own control (training phase performances compared to those obtained after surgery).

SWISS mice

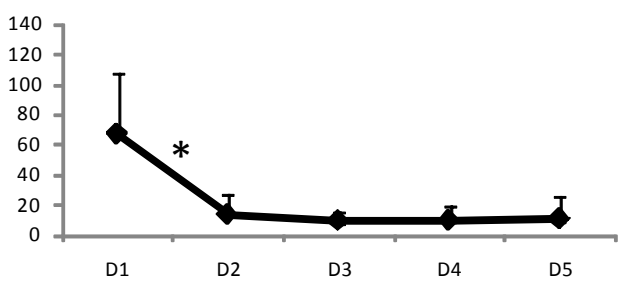

Young WISTAR rat

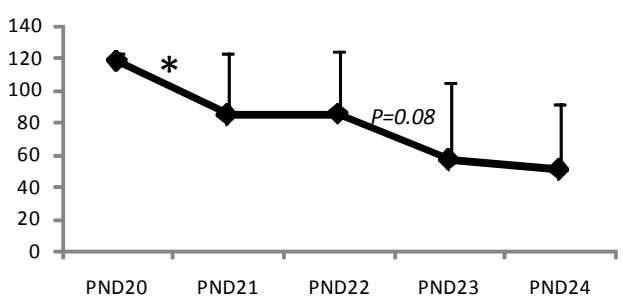

Marmoset

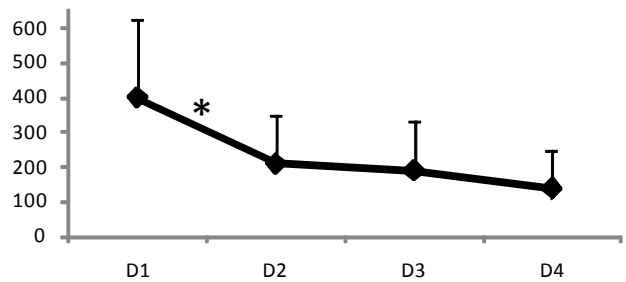

Adult SPRAGUE-DAWLEY rat

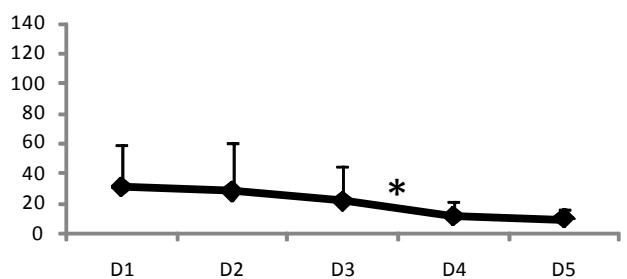

Fig. 1. Time to remove (s) an adhesive tape in four non-injured species during training. Data are expressed as mean $( \pm S D)$. In all species, ANOVA indicates a significant decrease with time $(p<0.0001$ in NMRI mice, $p=0.0005$ in marmosets, $p=0.0015$ in young Wistar rats, $\mathrm{p}<0.0001$ in adult Sprague Dawley rats). In most cases there is a decrease in performances between the first and the second day of training. 
Even though adult animals are used in most studies, we recently showed that it is possible to perform the adhesive removal test in young rats (from post-natal day 20) (Bouet et al., 2010) - enlarging thus the range of application of the test to perinatal ischemic stroke. In this case, pups were submitted to a brain injury at the age of 7-days old. Thus, training to the task before injury was not feasible making confounding training and deficits related to the injury. We observed the first day that young rats (20 days old) were unable to perform the task within the given time. Afterwards, sham animals showed better and better performances with time while lesioned animals stayed on the plateau level. This indicates that in particular cases, it is possible to obtain satisfactory data even without training. Our data showed that one week of training ( 1 trial per day) is sufficient for adult rats, mice and marmosets to reach a plateau level (Figure 1), whereas it is not the case for rat pups (PND20). This increased duration of training with young animals is partly due to the fact that rearing is required to remove adhesive and at this age, hindlegs weight bearing is still unstable. Of note, in marmosets, the stress of the procedure induces high values in the time to remove the adhesive the first days that is the reason why the maximal time given to the animal to perform the task is of $10 \mathrm{~min}$ ( $2 \mathrm{~min}$ for rodents). However, the plateau is reached by the $3^{\text {rd }}$ day of training.

\subsection{Body parts}

According to the species, adhesive removal is performed on different ways, related to the body parts on which adhesive tapes are placed, the parameters collected the place in which the test is performed... Concerning the body parts concerned, they often vary according to the location of the brain lesion and because of the differential sensitivity of different body parts of the species considered. For instance in rats, placing the adhesive on forepaws is the most efficient; indeed they do not really take care of adhesive placed on their back paws, probably because of weight support that induces a large stimulation of the plantar surface and strongly diminishes the discrimination. Additionally, sticking the adhesive tape on the forepaws of the animals will drive it to naturally remove the adhesive for its grooming. The same holds true for mice, in which adhesive test has been first described with positioning on the snout (Fleming et al., 2004), and then on forepaws (Bouet et al., 2009; Bouet et al., 2010; Bouet et al., 2007; Freret et al., 2009; Freret et al., 2006; Freret et al., 2006; Starkey et al., 2005), alike rats. In gerbils, and also in most of the studies performed in rats, the adhesive is placed on the wrist (Ishibashi et al., 2003). In primates, because of the role of hands in objects manipulation, adhesives are preferably placed around the feet (Annett et al., 1992; Freret et al., 2008; Marshall \&Ridley, 1996).

Consequently, while removing an adhesive pasted on the forepaws seems quite easy to do for rodents after training, it seems to be more tricky for marmosets. Figure 2 shows that while rodent stand on their hindpaws to remove the adhesive with the mouth, the marmoset has to stand on his bottom and raise the leg to bring the foot to the mouth. Such postures are rather close to the grooming postures for rodents, but they are more scarse in marmosets. This is one of the reasons that explain the increase in time to remove the adhesive for marmosets compared to rodents. The other reason is due to the fact that for marmosets the adhesive tapes enroll all the foot, making hard, therefore time-consuming to unroll. Such a long adhesive tape unrolling the limb of the animal can also be done in rodents, making it even more difficult to remove (Komotar et al., 2007). 


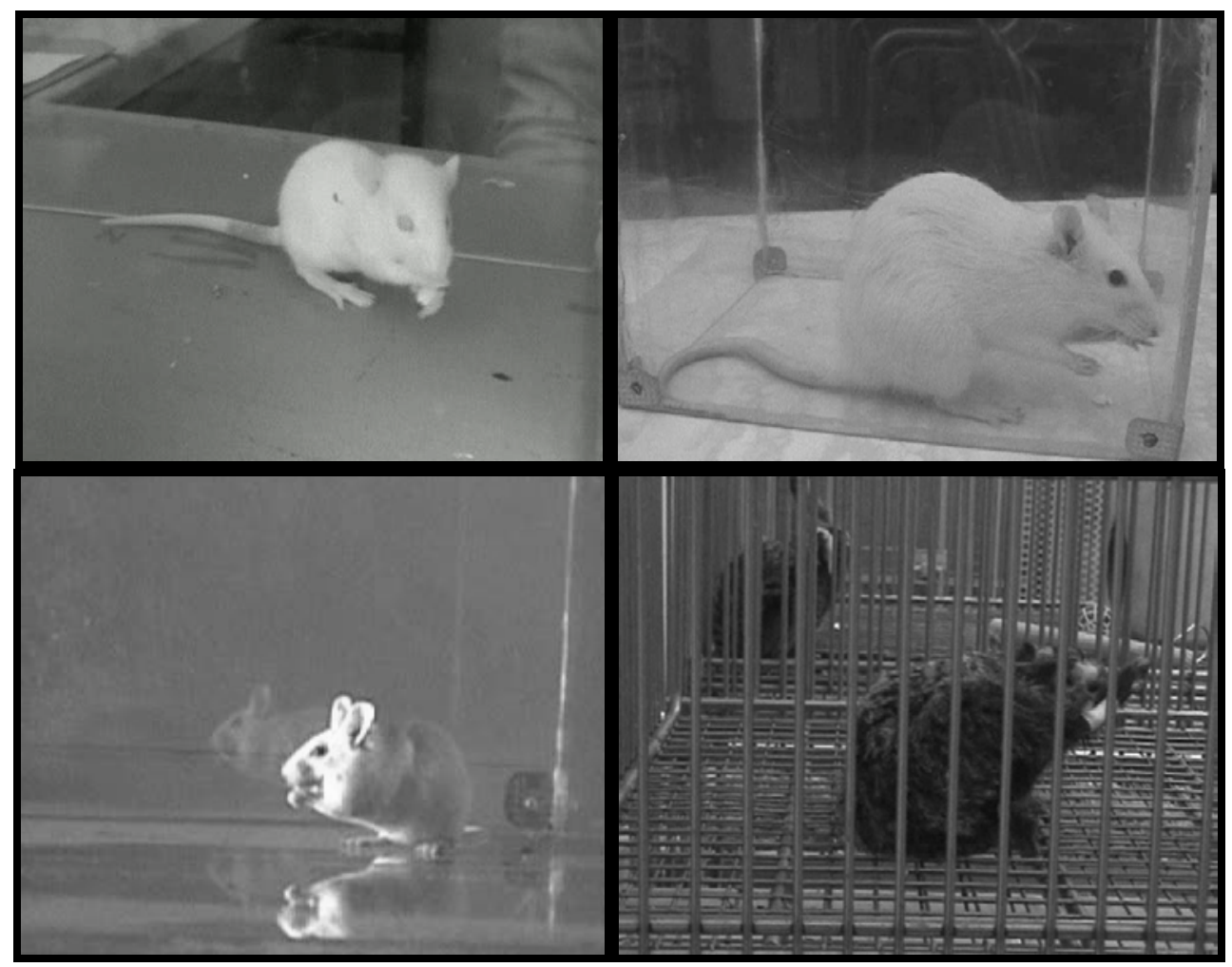

Fig. 2. Pictures depicting a young rat, an adult rat, a mouse and a marmoset being removing the adhesive tapes. All three species use the mouth to remove the adhesive. When very well trained, rats sometimes keep a three-feet posture to remove the tape. The training is very useful to render the animals accustomed to the procedure, and therefore to obtain homogenous data. When the training is not possible (for instance, if a brain injury is performed in young animals), the test is still possible, but the authors have to discuss the fact that the recovery and the learning of the task are mandatory overlapping.

\subsection{Adhesive tape size}

According to the species, the size of the tape has to be adapted and very tightly controlled. Indeed, Schallert and Whishaw (Schallert \&Whishaw, 1984) showed that the size of the adhesive could strongly influence the performances. In rats, Komotar et al. (Komotar et al., 2007) advised to use very large adhesive tapes and pasted them around the wrist, in order to make a kind of sleeve that cannot be removed for the duration of $30 \mathrm{~s}$ (the main parameter is the time the animals attempts to remove the adhesive). Most of the times, in rats and mice adhesive tape is small and pasted on the paw with the aim to cover the three pads, thenar and hypothenar (size: $1 \times 1 \mathrm{~cm}$ in rats (Freret et al., 2006); $0.3 \times 0.4 \mathrm{~cm}$ in mice (Bouet et al., 2007)). In primates, the tape has to be rectangular and should roll up the foot $(2 \times 4.5 \mathrm{~cm}$ in marmosets, (Freret et al., 2008)). Unavoidably, there are many differences between labs in the achievement of this test, and therefore the most important things to keep in mind is to 
always have a control group, always apply the adhesive with the same pressure on the right and left hand (an experimenter blind to the treatment is an obvious necessity), and always buy the adhesive tape from the same supplier (same brand). Our experience is that the sewed-adhesives used for bandage are the best (from Sogiphar, Urgo or BSN Medicals in France for example).

\subsection{Positioning of the adhesive}

Adhesive positioning requires animal contention to ensure a very good precision (Figure 3). Contention has to be performed as gently as possible, because any increase in stress can totally biases the results by increasing the time to perform the task, and this is true whatever the species. For the rat and the marmoset, the animal can be held by the torso by an experimenter, while the other experimenter places the tapes (Bouet et al., 2010; Freret et al., 2008; Freret et al., 2006). For the mouse and the gerbil, the animal can be held by the back skin in order to let the forepaw free (about the same way used to make intraperitoneal injection) (Bouet et al., 2009; Ishibashi et al., 2003). The experimenters have to get used with the contention before starting the experiments, by a previous training if necessary. The contention has to be gentle but firm. Rodents have to be held by the back skin as close to the neck as possible to maintain the head and thus prevent any biting. Once the animal is quietly held, the experimenter has to place an adhesive on each paw (alternating right and left between days and between animals), in a way that the pressure is identical on both paws and that the same skin portion is covered on both paws. To avoid the experimenter who is in charge to place the adhesive to be bite, it is important to maintain the foreleg as extended as possible by pulling the fingers before placing the adhesive tape. For rodents, the adhesive is rectangular or squared, and for marmosets it is rectangular and has to be placed around the foot in order to make a small overlap of the tissue.

The animal is then placed back in his own cage (without congeners) or in an experimental box (if this last is used, a habituation period has to be performed before positioning the adhesive by giving to the animal 1 or 2 minute free exploration of the box). Indeed, the test can be either performed in the home cage or in a testing box, depending on the animals housing conditions. If animals are single-housed, the first solution should be preferred, since it avoids any supplementary stress for animal. As a contrary, carrying out the adhesive removal in a testing box rather than in the home cage, should be preferred when animal are group-housed to avoid interaction between individuals that can alter performances. Nevertheless, in the former case, a habituation period to the testing box should be respected.

\subsection{Collecting the data}

Once the animal is placed back in the cage with the two adhesive pasted on the paws, four different values have to be collected: time to contact and time to remove the adhesive for each paw.

- Tactile responses are measured by time to initial contact, which is related to the time taken by the animal to react to the presence of each adhesive. It is often considered as a sign of sensory system stimulation. The response of the animal to this stimulation is generally a shake of the paw of a touch with the mouth. For marmoset it can also be a scratching of the leg on the side of the adhesive.

- Time to remove is related to the time needed to completely take away each adhesive. Rodents as well as marmosets generally remove the adhesive with their mouth. Rodents 
usually take a small piece between teeth and remove it in one movement (Figure 2), while marmosets often try several times to remove it by biting. The time to remove reflects as well as sensory and motor abilities, since it requires a correct dexterity.

\section{Mice}

Rats
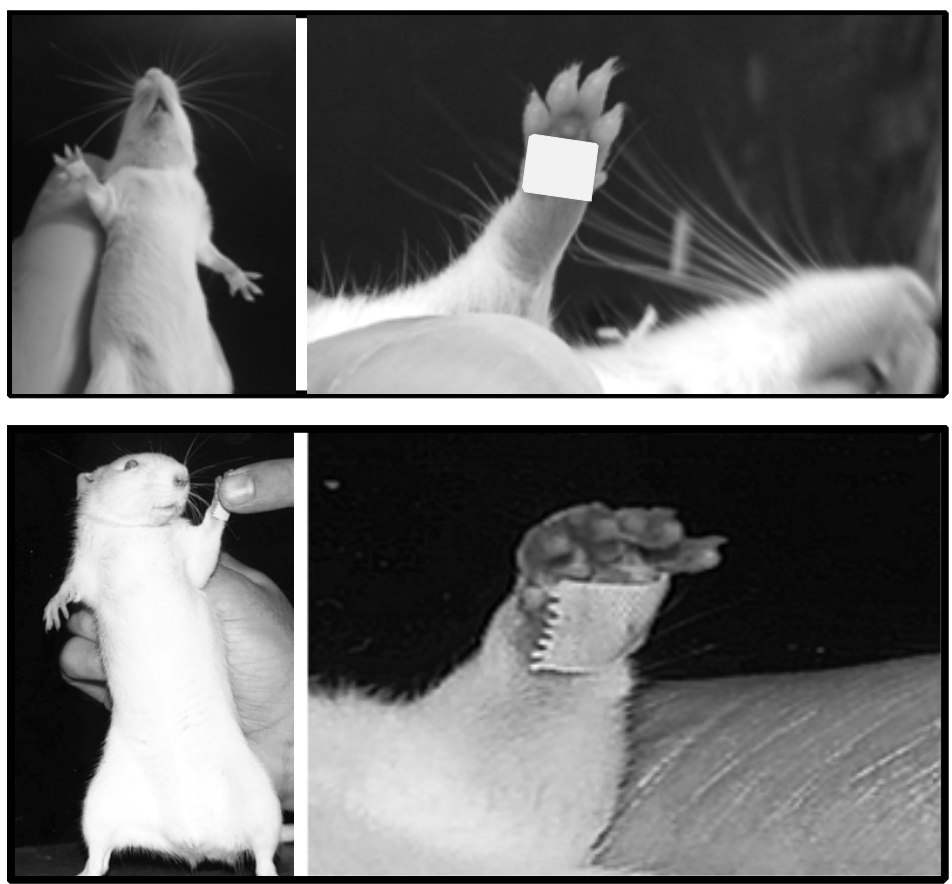

\section{Marmosets}

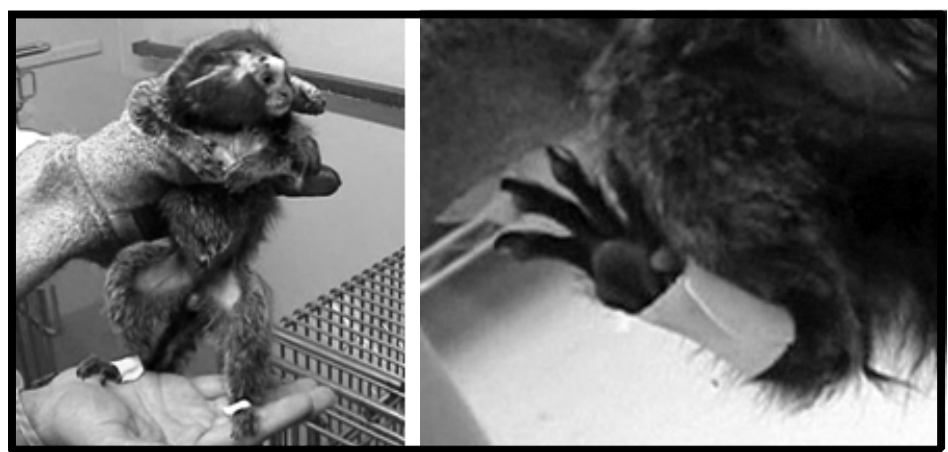

Fig. 3. Positioning of the adhesive tapes in mice, rats and marmosets. The piece of adhesive has to be positioned on fore paws for mice and rats, and on hind paws in marmosets (alternation between left and right for the first positioning and between trials should be achieved).

Overall, time to contact and time to remove crudely separate out sensory versus motor deficits (Schaar et al., 2010). On a side point, it should be noted that the adhesive removal test can also be used to measure animals sensory asymmetries. The magnitude of sensory 
asymmetry is measured by adjusting the ratio of the size of the adhesive tapes on each limb. This test can thus reveal asymmetrical biases in stimulus-directed activity after focal ischemia (Schallert et al., 2000).

\subsection{Analyzing of the data collected}

Statistical analyses have to be conducted on several parameters to check first some possible discrepancies but also to measure the intensity of the deficits and of the recovery. The first important point is to check, before any brain injury, if there is no initial asymmetry between left and right side. Indeed, it has been showed that for certain sensorimotor tasks, rodents could display an important asymmetry (Bulman-Fleming et al., 1997). The statistical analysis will therefore compare contralateral and ipsilateral removal and contact times. The second point concerns the appraisal of the deficit. To this, two different possibilities exist: data obtained after the injury could be compared to those obtained before the insult (this is also important in sham-operated animals to assess if surgery in itself induces or not any deficit). In this case, it is interesting to express data in percentages compared to pre-surgery. Otherwise, comparisons could also concern data obtained on the controlateral versus ipsilateral side. This allows comparing two measurements performed on the same animals within the same session. Moreover, this allows calculating an asymmetry index (contralateral - ipsilateral time).

As regards to number of animals per group, previous studies from the literature have proved that even a small number of animals per group allow to show statistical difference in the adhesive removal test (Freret et al., 2006; Zhang et al., 2011).

\section{Deficits in adhesive removal test after stroke in rodents and marmosets}

The adhesive-removal test is classically used to detect forepaw somatosensory - time to contact - and sensorimotor - time to remove - deficits that are not attributable to postural bias (Schallert et al., 1983). Its sensitivity to ischemia-induced deficits has already been reported in the literature (Modo et al., 2000) for every species in which it has been developed, and in most experimental models of cerebral ischemia. For best comparisons between species, most of the results presented here are related to the intra-luminal model of cerebral ischemia. This model has been chosen to illustrate our purpose, because it concerns the middle cerebral artery (MCA) - the most frequently affected artery in stroke patients, because duration of occlusion can be modified, but mostly because a reperfusion event is feasible and thus this closely mimics what happens in patients. In this model, the site of the occlusion of the MCA (MCAo) could either be proximal (close to the origin of the artery, p-MCAo) or distal (after the lenticulostriate branches, d-MCAo). In case of p-MCAo, behavioral deficits in the adhesive removal test are readily observable - even in mice, a species in which subtle behavioral changes are particularly difficult to detect. However, p-MCAo is not representative of all clinical situations because it leads to brain infarctions that are relatively larger than those observed in human stroke. As it induces smaller infarct, d-MCAo is more relevant to those clinical situations. Behavioral alterations after d-MCAo have been largely explored in rats or in marmosets, but, unfortunately, most of the studies using this model in mice considered time points early after surgery and mostly addressed lesion size. Only motor coordination difficulties, attentional deficits and a low increase in eye movement during the dark phase of sleep have been reported in mice (Baumann et al., 2006; Guegan et 
al., 2006; van Lookeren Campagne et al., 1999). The reason for this lack in literature is that long-term behavioral deficits (i.e., several weeks after surgery) are difficult to detect in mice. Iadecola and colleagues even explained that they had to proximally occlude the artery "because dMCA occlusion produced no neurological deficits" in the mouse (Iadecola et al., 1997). This is presumably because of the low sensitivity of the behavioral testing available in the literature. Nevertheless, mice are of real interest in experimental studies because of their low cost and possible transgenic alterations. To further argue for the usefulness of the adhesive removal test, some data illustrating deficits observed in mice after d-MCAo are presented below (see figure 4). Surgical procedures used to induce stroke are described in Bouet et al., 2007, Bouet et al., 2010, Freret et al., 2008, Freret et al., 2009.

\subsection{Expected results after cerebral ischemia across animals' species}

As a rule, a highly significant impairment on the contralateral side is commonly observed, whereas the deficit is usually more or less important on the ipsilateral side. Besides, the contralateral deficit is often long-lasting with a delay that will vary according to the species considered and the duration of the occlusion of the middle cerebral artery, i.e. the severity of the injury (Figure 4). As regards to spontaneous recovery phenomenon, the ipsilateral deficit often disappears during the first stage after the insult. Quite a contrary, improvement of performances on the contralateral side is not always observed, once again according to the design of the study. This discrepancy of rate of recovery between ipsi- and controlateral side makes the adhesive removal test an efficient tool for assessing the kinetic of functional outcome after cerebral ischemia (acute and long-term phase). Thus, this task may be suitable for assessing both neuroprotective therapies which target early intervention as well as those aimed at the prevention of delayed damage and therapies which promote regeneration.

In rats, while no somatosensory impairment is observed after a 30-min duration of occlusion of the middle cerebral artery (MCAo), 60-min of occlusion induces a bilateral and long lasting deficit, as reflected by an increased time to contact (Figure 4; (Freret et al., 2006)). This deficit tends however to partially recover over time, as attested by the decreasing slope of the time to contact curves. To ensure any potent ischemia-induced somatosensory asymmetry, an index giving the difference between ipsi- and controlateral performances can be calculated (see Freret et al., 2006; Bouet et al., 2007). Thus, comparing contro- and ipsilateral side, it should be note that the rats display a preference towards the ipsilateral, thus non paretic side.

As regards to motor abilities estimated by the time to remove the adhesive, a unilateral and transient deficit can be observed after a 30-min MCAo (up to 1 week after surgery). By contrast, 60-min MCAo will drastically impact on motor functions; a bilateral motor coordination deficit - albeit only transient on the ipsilateral side - can be observed. This long-lasting deficit on the contralateral side reflects a failure to respond to a novel tactile stimulus.

In mice, considering a 60-min duration of occlusion of the MCA (p-MCAo), a bilateral somatensory deficit can be highlighted - as attested by the increased time to contact the adhesives (Bouet et al., 2009; Bouet et al., 2007). Besides, as regards to the time to remove the adhesives, a bilateral impairment can be observed after ischemia. Our group has observed that this deficit in motor coordination appears to be long lasting, longer time to remove the adhesive on the contralateral side of the lesion, since it is still observable up to 6 weeks after surgery (unpublished data). 
Time to contact contralateral adhesive after stroke (\% pre-surgery)
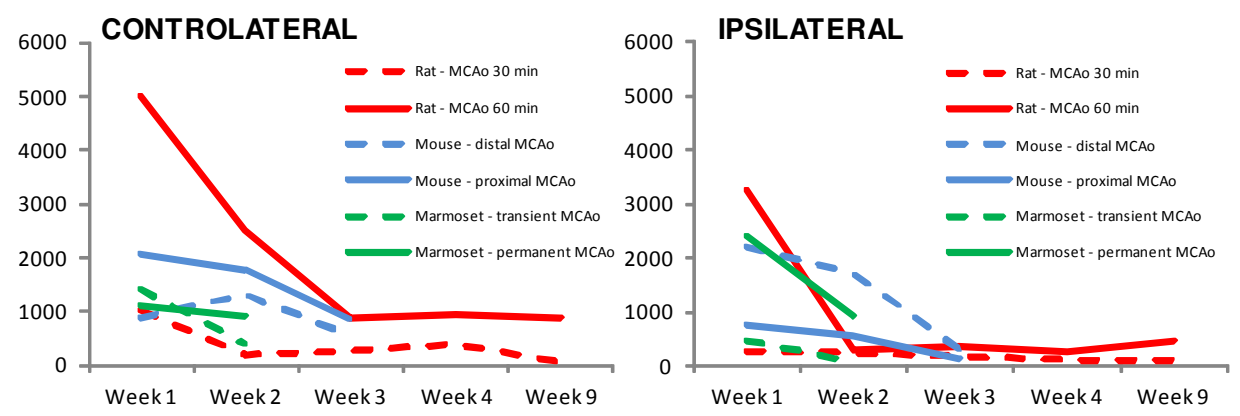

Time to remove contralateral adhesive after stroke (\% pre-surgery)
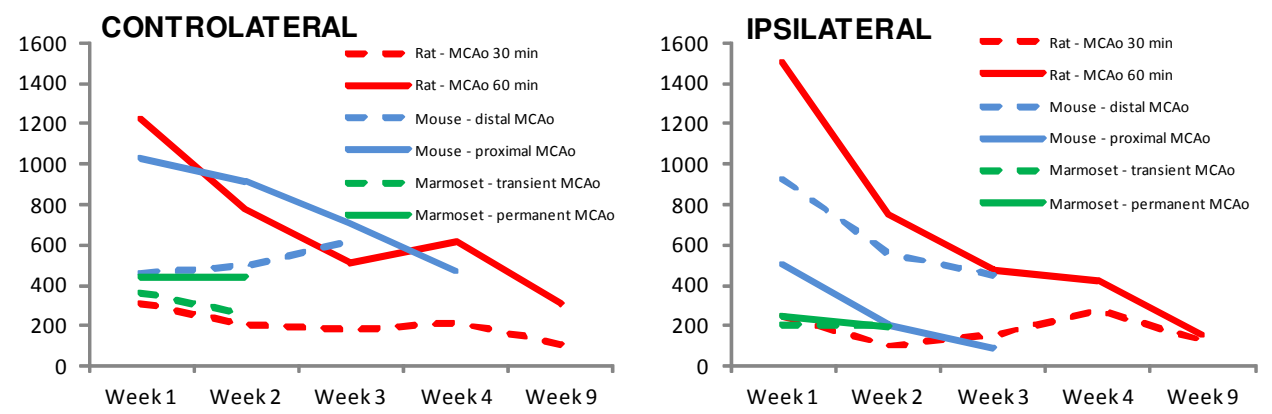

Fig. 4. Adhesive removal performances in rat, mouse and marmoset after stroke (\% pre-surgical values). Mean percentage of time to contact the adhesive tapes (upper graphs), positioned on the controlateral side (left) and on the ipsilateral side (right). Time to remove the adhesive tapes (lower graphs), positioned on the controlateral side (left) and on the ipsilateral side (right). All stroke models presented concern the occlusion the middle cerebral artery (MCAo). For rats, stroke was induced by the intraluminal occlusion of the MCA (30 or $60 \mathrm{~min}$ ). For mice, MCAo was occluded either by distal permanent electrocoagulation or by intraluminal occlusion (60 min - proximal). In marmosets, MCA was occluded by intraluminal approach either permanently of transiently $(3 \mathrm{~h})$. Each point represents the mean of three trials performed on three consecutive days.

Take a look now at the impairments associated to relatively small brain lesion (d-MCAo), adhesive removal test is very useful to detect functional contralateral deficits, even 3 weeks after surgery (Freret et al., 2009). Whereas alteration in the somatosensory perception of the contralateral adhesive (time to contact) partially recovers within 3 weeks after ischemia, the sensorimotor contralateral impairment (time to remove) is still strongly present on the $3^{\text {rd }}$ week after surgery.

In marmoset, an early bilateral somatosensory deficit (time to contact) (Freret et al., 2009) is induced by either transient $(3 \mathrm{~h})$ or permanent proximal MCAo. This deficit is often transient 
on the ipsilateral side of the lesion and might be due, at least partly, to dizziness of the animal due to surgery and/or the anesthesia. With respect to the time to remove the adhesive, a bilateral motor coordination deficit is observed whatever the duration of the occlusion. Of note, it has been demonstrated in this same model of cerebral ischemia a spontaneous functional recovery on the ipsilateral side, while controlateral time to remove the adhesive remains hardly affected up to 4 weeks after surgery (Bihel et al.).

\subsection{Correlations between brain histological damage and deficit in the adhesive removal test}

Correlations between the cortical and striatal histological lesions and the ischemia-induced behavioral impairments in the adhesive removal test have been well investigated in the literature, mostly in rodents (Grabowski et al., 1991; Hudzik et al., 2000; Hunter et al., 1998; Rogers et al., 1997; Virley et al., 2000). We and other authors have demonstrated a close correlation between contralateral contact and removal latencies on this task and abnormal changes in the ipsilateral caudate putamen, lower parietal cortex and forelimb cortex following transient MCAo (30; 60 or 90 min; see Figure 5). Each of these regions of interest contributed to functional impairments on this task across an extended time course (up to several months post-ischemia) (Virley et al., 2000). As regards to the final cortical damage, it seems particularly correlated to both transient and long-lasting sensorimotor deficits measured by adhesive removal test. Conversely, the final striatal lesion appears to be consistently related to the adhesive removal motor deficits (time to remove) (Freret et al., 2006).

\section{Rodents}

\section{Distal MCAO}

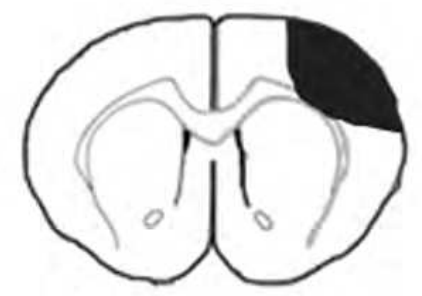

\section{Proximal MCAo}

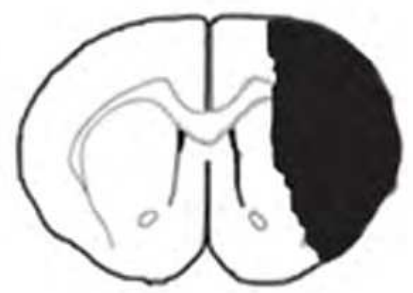

Marmoset
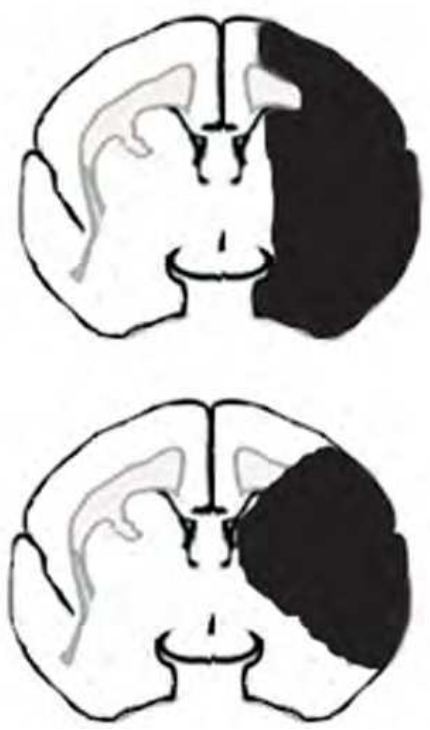

Fig. 5. Schemes showing extent of the lesion after distal and proximal models of Middle Cerebral Artery occlusion (MCAo) in rodents and marmoset. Distal MCAo refers to the occlusion of the artery in its distal portion, i.e. after lenticulostriate branches (usually 
obtained by electrocoagulation or administration of pharmacological agent, e.g. endothelin-1 (Virley et al., 2004)), while proximal MCAo refers to an occlusion at the origin of the artery (intraluminal approach). Extent of the lesion has been drawn from our data and literature. Of note, these schemes are not fully representative of all experimental models of middle cerebral artery ischemia, but give an overview of most frequently obtained brain lesion.

On a side point, the potential relationship between impairments in the adhesive removal test and brain regions that undergo secondary degeneration has been less investigated so far. Indeed, ischemia is known to cause secondary degeneration in non-ischemic remote brain areas such as the ipsilateral thalamus (mostly the ventroposteromedial and ventroposterolateral nuclei (Iizuka et al., 1990)) as well as in the substantia nigra (Tamura et al., 1990). These regions that do not belong to the territory of the MCA, are connected to the primary lesion site. As regards to secondary thalamus shrinkage, retrograde as well as anterograde degeneration due, respectively, to cortical lesion (Iizuka et al., 1990) (Wallerian) and to basal ganglia lesion and/or extensive vasogenic edema (Dihne et al., 2002) are thought to be responsible. Whether the extent of these degenerative changes is or not directly correlated to the size of primary lesion is still under controversy (Freret et al., 2006; Iizuka et al., 1990). No matter how, in a rat stroke model, the final thalamic atrophy appears to be correlated with ischemia-induced deficits observed in the adhesive-removal test (especially its sensory component) (Freret et al., 2006). This correlation suggests that the thalamus makes, albeit moderately, a significant contribution to the ischemia-induced longlasting somatosensory (ipsilateral bias) and sensorimotor deficits (dexterity alteration). These findings are in agreement with the known involvement of thalamic ventroposterior nuclei in the somatosensory pathways and sensory processing (Tracey \&Waite, 1995). In addition, correlations appeared at the late chronic stage (i.e., several weeks after ischemic insult, which is consistent with the delay of thalamic shrinkage development; (Fujie et al., 1990; Jones \&Schallert, 1992). Regarding substantia nigra, damage to this brain structure is also correlated with contralateral deficits in the adhesive removal test (Virley et al., 2000).

Finally, talking about correlations between impairments in the adhesive removal test and brain damages in experimental models of focal ischemia, one must keep in mind that although the presence of such a correlation would make the interpretation of performances in this behavioral test easier, the absence of a linear relationship between both parameters does not mean the absence of involvement of the structure in the behavioral task. Indeed, one can imagine that beyond a single and direct relationship between one or several brain structures and the behavioral performances, those latter might depend upon the integrity of a cerebral network that can be influenced by others brain structures. If so, a lesion limited only to this cerebral network or to the related brain structures will have consequences on behavioral performances; even though the one or the other remains unaffected.

\subsection{Sensitivity of the adhesive removal test to pharmacological treatment}

In line with this consideration of a relationship between impairments in the adhesive removal test and brain damages in experimental models of focal ischemia, our group have demonstrated that a delayed and chronic administration of deferoxamine (an iron chelator) reduces the secondary thalamic atrophy and improves functional recovery in the adhesive removal test after focal ischemia in the rat (Freret et al., 2006). Similarly, a neuroprotective effect of D-JNKi (a peptide inhibitor of JNK (c-Jun-N-terminal kinase)) have been highlighted and corroborated with adhesive removal improvement in the rat (Esneault et 
al., 2008). This test has also proven to be useful for cellular therapies (Minnerup et al., 2011; Shen et al., 2007), or other pharmacological approaches (testosterone injection - (Morris et al., 2010)), Thymosin beta4 I - (Pan et al., 2005)). In mice, Rehni et al., 2007 (Rehni et al., 2007) showed that intracerebroventricular administration of stem cells derived from amniotic fluid is beneficial for adhesive removal after 60-min MCAo in mice. In marmosets, till now only few studies have assessed the effects of pharmacological agents on adhesive removal test after stroke, but much on Parkinson's disease (Annett et al., 1994).

Overall, those results argue in favor of the use of the adhesive removal test to accurately assess the effects of pharmacological agents on functional outcome. Indeed, this task is suitable for assessing both neuroprotective therapies which target early intervention as well as those aimed at the prevention of delayed damage and therapies which promote regeneration.

\section{General overview and limitations of the adhesive removal test}

Data reported here show that adhesive removal test $i$. can be easily performed in most species used in experimental research ii. is powerful to point out functional deficits on a long-term duration after brain injury. This is a particularly interesting point because it allows assessing efficiency of new therapeutic strategies. Those deficits are in human patients those for which no real efficient therapy exists, excepted rehabilitative strategies like kinesiotherapy. Measuring their importance in animals give models to go further in researches on brain injury recovery.

Contact and removal times give complementary information about the nervous system deficiency. Although the measurement of contact time is not a perfect reflect of sensory system functioning, because for obvious reasons it is related to a motor action (shaking paw, bringing paw to the mouth), it reflects nevertheless a sensory system stimulation. The animal feels or not that something is stuck on its paw and reacts by a movement. From experimental data obtained with adhesive removal, it is not possible to distinguish if the deficit in contact time is related to a primary somatosensory dysfunction (Ward et al., 1997), a sensory hemi-neglect (related to attentional deficit of a body part and involving striatum (Heilman et al., 2000; Reep et al., 2004)), or a tactile extinction phenomenon (related to bilateral stimulation (Schallert \&Whishaw, 1984)).

Removal of the tape is a rather tricky task for all the animals presented here because it is not per se a natural situation. Rodents and marmosets use their mouth to do it, and amazingly after stroke rats often hold their deficient paw with the intact one to bring it to the mouth as if they were not able to maintain at the right position the deficient/controlateral hand.

The data presented here show that for equivalent brain lesions (parietal cortex and striatum affected by intraluminal model of stroke) all species tested display long-lasting deficits in removal time, at least at the dates tested, which is however rather long compared to delays usually assessed in stroke investigations. Time to contact the contralateral adhesive is also increased for few weeks after stroke, and comes back to normal levels for the less severe brain injury, while it stays abnormal for more severe injuries. It is important to note that the severity of the deficit is quite well related with the extent of the lesion, but also to the structures affected. When the parietal cortex alone is affected (30-min MCAo in rat, distal model in mouse), the deficit is less severe than when other structures are also involved in the lesioned area. Indeed, when the striatum is also affected (60-min MCAo in rat, proximal 
model in mouse, both models in marmoset), the recovery is even longer and most of the time not complete within the delays tested.

Mechanisms implied in the recovery of the ability to remove the adhesive tape are not well determined but some of them have to be considered: structural modifications are undoubted (sprouting, synaptogenesis,..), functional modifications (synaptic plasticity, use of brain areas close to the lesion site to fulfill the roles of destroyed tissue...), sensory substitution (increasing role of deep cutaneous modality to replace for the superficial one...), or even behavioral strategies (as for instance, the rat holding the deficient paw with the intact paw to bring it to its mouth).

\subsection{Sensory hemi-neglect or tactile extinction syndrome}

In rats, a 60-min MCAo induces a long-lasting motor coordination deficit on the contralateral side, reflecting a failure to respond to a novel tactile stimulus. Whether this phenomenon reflects either a primary somatosensory dysfunction (Ward et al., 1997), a truly sensory hemi-neglect (i.e., an attention deficit, as it is classically invoked in the clinic; (Heilman et al., 2000)), or a tactile extinction syndrome (i.e., an interhemispheric perceptual interaction between both stimuli; (Schallert \&Whishaw, 1984) is hard to say. Indeed, such duration of occlusion induces a lesion that includes the primary sensory cortical area (S1FL) and, in a secondary degenerative manner, part of the thalamus. These two regions are involved in the somatosensory information processing. On the other hand, it has been shown that unilateral lesions of the posterior parietal cortex and of the dorsocentral striatum (i.e., two structures that are at least partly affected by ischemia) result in multimodal neglect in the rat (Reep et al., 2004). Clinical studies on healthy subjects have reported that the vigilance component of attention to sensory stimuli involves, at least in part, the parietal cortex (Pardo et al., 1991). Thus, a lesion of the parietal cortex could also contribute to a sensory neglect syndrome in the rat after ischemia. An alteration of the cortical- basalganglia-thalamic network could lead to a sensory neglect syndrome in the rat. Nevertheless, a tactile extinction syndrome occurs frequently in stroke patients with right cerebral hemisphere damage (Rose et al., 1994), and the presence of neglect is not only debilitating for patient's ability for independent daily life but is also a significant predictor of poor outcome for recovery from hemiplegia in stroke patients (Denes et al., 1982). The longlasting correlation described between the contralateral time to contact and the final cortical damage (Freret et al., 2006) reinforces the idea that this structure makes significant contribution to the sensory impairment observed after ischemia (Virley et al., 2000).

\subsection{Facial and/or limb impairments}

After d-MCAo in mice, even though the extension of the lesion is relatively limited, the adhesive removal test is efficient to highlight sensorimotor contralateral impairment (time to removal) 3 weeks after the surgery. This deficit may combine both sensory feedback alterations and motor coordination that are not attributable to postural bias (Schallert et al., 1983). Given the spatial distribution of the cerebral lesion (Figure 5), this impairment might also reflect in this case a face-related somatososensory perception alteration (i.e., a difficulty for the animal to sense the adhesive with its ipsilateral whiskers when it approaches the tape to his face and/or with its tongue when he licks the adhesive in order to facilitate the removal) rather than a forelimb motor or sensory alteration (because the related brain region, i.e., S1 FL, is mainly spared by ischemia). This hypothesis is in accordance with a 
study reporting the usefulness of the tongue protrusion test in a rat model of proximal cerebral ischemia (Gulyaeva et al., 2003). Furthermore, it suggests that permanent distal stroke could be a relevant model of oral and facial impairments, which are also tremendous problems in human stroke.

\section{Conclusion}

To conclude on the usefulness of the adhesive removal test, one must admit that this test:

1. is relevant because it was developed in relation to clinical evaluations,

2. is an accurate test to assess somatosensory and motor dysfunctions, even if they are very tiny,

3. is useful to assess recovery, since it is capable of measuring long-lasting deficits,

4. allows for longitudinal studies through adaptation of the size of the adhesive tape according to the age of the individual tested. Indeed, as previously described, we adapted the adhesive removal test to in 20 days old rat pups by reducing the size of the adhesive tape.

5. Finally, the adhesive removal test allows for interspecies comparison (marmosets, rats, dogs and mice), as strongly advised by the expert committees for preclinical studies.

\section{References}

Annett LE, Martel FL, Rogers DC, Ridley RM, Baker HF, Dunnett SB. Behavioral assessment of the effects of embryonic nigral grafts in marmosets with unilateral 6-OHDA lesions of the nigrostriatal pathway. Exp Neurol 1994;125(2):228-46.

Annett LE, Rogers DC, Hernandez TD, Dunnett SB. Behavioural analysis of unilateral monoamine depletion in the marmoset. Brain 1992;115 ( Pt 3):825-56.

Baumann CR, Kilic E, Petit B, Werth E, Hermann DM, Tafti M, Bassetti CL. Sleep EEG changes after middle cerebral artery infarcts in mice: different effects of striatal and cortical lesions. Sleep 2006;29(10):1339-44.

Bihel E, Pro-Sistiaga P, Letourneur A, Toutain J, Saulnier R, Insausti R, Bernaudin M, Roussel S, Touzani O. Permanent or transient chronic ischemic stroke in the nonhuman primate: behavioral, neuroimaging, histological, and immunohistochemical investigations. J Cereb Blood Flow Metab;30(2):273-85.

Bouet V, Boulouard M, Toutain J, Divoux D, Bernaudin M, Schumann-Bard P, Freret T. The adhesive removal test: a sensitive method to assess sensorimotor deficits in mice. Nat Protoc 2009;4(10):1560-4.

Bouet V, Freret T, Ankri S, Bezault M, Renolleau S, Boulouard M, Jacotot E, Chauvier D, Schumann-Bard P. Predicting sensorimotor and memory deficits after neonatal ischemic stroke with reperfusion in the rat. Behav Brain Res 2010.

Bouet V, Freret T, Toutain J, Divoux D, Boulouard M, Schumann-Bard P. Sensorimotor and cognitive deficits after transient middle cerebral artery occlusion in the mouse. Exp Neurol 2007;203(2):555-67.

Bulman-Fleming MB, Bryden MP, Rogers TT. Mouse paw preference: effects of variations in testing protocol. Behav Brain Res 1997;86(1):79-87.

Denes G, Semenza C, Stoppa E, Lis A. Unilateral spatial neglect and recovery from hemiplegia: a follow-up study. Brain 1982;105 (Pt 3):543-52. 
Dihne M, Grommes C, Lutzenburg M, Witte OW, Block F. Different mechanisms of secondary neuronal damage in thalamic nuclei after focal cerebral ischemia in rats. Stroke 2002;33(12):3006-11.

Esneault E, Castagne V, Moser P, Bonny C, Bernaudin M. D-JNKi, a peptide inhibitor of cJun N-terminal kinase, promotes functional recovery after transient focal cerebral ischemia in rats. Neuroscience 2008;152(2):308-20.

Fleming SM, Salcedo J, Fernagut PO, Rockenstein E, Masliah E, Levine MS, Chesselet MF. Early and progressive sensorimotor anomalies in mice overexpressing wild-type human alpha-synuclein. J Neurosci 2004;24(42):9434-40.

Freret T, Bouet V, Leconte C, Roussel S, Chazalviel L, Divoux D, Schumann-Bard P, Boulouard M. Behavioral deficits after distal focal cerebral ischemia in mice: Usefulness of adhesive removal test. Behav Neurosci 2009;123(1):224-30.

Freret T, Bouet V, Toutain J, Saulnier R, Pro-Sistiaga P, Bihel E, Mackenzie ET, Roussel S, Schumann-Bard $\mathrm{P}$, Touzani O. Intraluminal thread model of focal stroke in the nonhuman primate. J Cereb Blood Flow Metab 2008;28(4):786-96.

Freret T, Chazalviel L, Roussel S, Bernaudin M, Schumann-Bard P, Boulouard M. Long-term functional outcome following transient middle cerebral artery occlusion in the rat Correlation between brain damage and behavioral impairment. Behav Neurosci 2006:in press.

Freret T, Valable S, Chazalviel L, Saulnier R, Mackenzie ET, Petit E, Bernaudin M, Boulouard M, Schumann-Bard P. Delayed administration of deferoxamine reduces brain damage and promotes functional recovery after transient focal cerebral ischemia in the rat. Eur J Neurosci 2006;23(7):1757-65.

Fujie W, Kirino T, Tomukai N, Iwasawa T, Tamura A. Progressive shrinkage of the thalamus following middle cerebral artery occlusion in rats. Stroke 1990;21(10):1485-8.

Grabowski M, Nordborg C, Johansson BB. Sensorimotor performance and rotation correlate to lesion size in right but not left hemisphere brain infarcts in the spontaneously hypertensive rat. Brain Res 1991;547(2):249-57.

Guegan C, Braudeau J, Couriaud C, Dietz GP, Lacombe P, Bahr M, Nosten-Bertrand M, Onteniente B. PTD-XIAP protects against cerebral ischemia by anti-apoptotic and transcriptional regulatory mechanisms. Neurobiol Dis 2006;22(1):177-86.

Gulyaeva N, Thompson C, Shinohara N, Lazareva N, Onufriev M, Stepanichev M, Moiseeva Y, Fliss H, Hakim AM. Tongue protrusion: a simple test for neurological recovery in rats following focal cerebral ischemia. J Neurosci Methods 2003;125(1-2):183-93.

Heilman KM, Valenstein E, Watson RT. Neglect and related disorders. Semin Neurol 2000;20(4):463-70.

Hudzik TJ, Borrelli A, Bialobok P, Widzowski D, Sydserff S, Howell A, Gendron P, Corbett D, Miller J, Palmer GC. Long-term functional end points following middle cerebral artery occlusion in the rat. Pharmacol Biochem Behav 2000;65(3):553-62.

Hunter AJ, Mackay KB, Rogers DC. To what extent have functional studies of ischaemia in animals been useful in the assessment of potential neuroprotective agents? Trends Pharmacol Sci 1998;19(2):59-66.

Iadecola C, Zhang F, Casey R, Nagayama M, Ross ME. Delayed reduction of ischemic brain injury and neurological deficits in mice lacking the inducible nitric oxide synthase gene. J Neurosci 1997;17(23):9157-64. 
Iizuka H, Sakatani K, Young W. Neural damage in the rat thalamus after cortical infarcts. Stroke 1990;21(5):790-4.

Ishibashi S, Kuroiwa T, Endo S, Okeda R, Mizusawa H. Neurological dysfunctions versus regional infarction volume after focal ischemia in Mongolian gerbils. Stroke 2003;34(6):1501-6.

Jones TA, Schallert T. Subcortical deterioration after cortical damage: effects of diazepam and relation to recovery of function. Behav Brain Res 1992;51(1):1-13.

Komotar RJ, Kim GH, Sughrue ME, Otten ML, Rynkowski MA, Kellner CP, Hahn DK, Merkow MB, Garrett MC, Starke RM, Connolly ES. Neurologic assessment of somatosensory dysfunction following an experimental rodent model of cerebral ischemia. Nat Protoc 2007;2(10):2345-7.

Marshall JW, Ridley RM. Assessment of functional impairment following permanent middle cerebral artery occlusion in a non-human primate species. Neurodegeneration 1996;5(3):275-86.

Minnerup J, Kim JB, Schmidt A, Diederich K, Bauer H, Schilling M, Strecker JK, Ringelstein EB, Sommer C, Scholer HR, Schabitz WR. Effects of neural progenitor cells on sensorimotor recovery and endogenous repair mechanisms after photothrombotic stroke. Stroke 2011;42(6):1757-63.

Modo M, Stroemer RP, Tang E, Veizovic T, Sowniski P, Hodges H. Neurological sequelae and long-term behavioural assessment of rats with transient middle cerebral artery occlusion. J Neurosci Methods 2000;104(1):99-109.

Morris DC, Chopp M, Zhang L, Lu M, Zhang ZG. Thymosin beta4 improves functional neurological outcome in a rat model of embolic stroke. Neuroscience 2010;169(2):674-82.

Pan Y, Zhang H, Acharya AB, Patrick PH, Oliver D, Morley JE. Effect of testosterone on functional recovery in a castrate male rat stroke model. Brain Res 2005;1043(12):195-204.

Pardo JV, Fox PT, Raichle ME. Localization of a human system for sustained attention by positron emission tomography. Nature 1991;349(6304):61-4.

Quaranta A, Siniscalchi M, Frate A, Vallortigara G. Paw preference in dogs: relations between lateralised behaviour and immunity. Behav Brain Res 2004;153(2):521-5.

Reep RL, Corwin JV, Cheatwood JL, Van Vleet TM, Heilman KM, Watson RT. A rodent model for investigating the neurobiology of contralateral neglect. Cogn Behav Neurol 2004;17(4):191-4.

Rehni AK, Singh N, Jaggi AS, Singh M. Amniotic fluid derived stem cells ameliorate focal cerebral ischaemia-reperfusion injury induced behavioural deficits in mice. Behav Brain Res 2007;183(1):95-100.

Rogers DC, Campbell CA, Stretton JL, Mackay KB. Correlation between motor impairment and infarct volume after permanent and transient middle cerebral artery occlusion in the rat. Stroke 1997;28(10):2060-5; discussion 6.

Rose L, Bakal DA, Fung TS, Farn P, Weaver LE. Tactile extinction and functional status after stroke. A preliminary investigation. Stroke 1994;25(10):1973-6.

Schaar KL, Brenneman MM, Savitz SI. Functional assessments in the rodent stroke model. Exp Transl Stroke Med 2010;2(1):13.

Schallert T, Fleming SM, Leasure JL, Tillerson JL, Bland ST. CNS plasticity and assessment of forelimb sensorimotor outcome in unilateral rat models of stroke, cortical 
ablation, parkinsonism and spinal cord injury. Neuropharmacology 2000;39(5):77787.

Schallert T, Upchurch M, Lobaugh N, Farrar SB, Spirduso WW, Gilliam P, Vaughn D, Wilcox RE. Tactile extinction: distinguishing between sensorimotor and motor asymmetries in rats with unilateral nigrostriatal damage. Pharmacol Biochem Behav 1982;16(3):455-62.

Schallert T, Upchurch M, Wilcox RE, Vaughn DM. Posture-independent sensorimotor analysis of inter-hemispheric receptor asymmetries in neostriatum. Pharmacol Biochem Behav 1983;18(5):753-9.

Schallert T, Whishaw IQ. Bilateral cutaneous stimulation of the somatosensory system in hemidecorticate rats. Behav Neurosci 1984;98(3):518-40.

Shen LH, Li Y, Chen J, Cui Y, Zhang C, Kapke A, Lu M, Savant-Bhonsale S, Chopp M. Oneyear follow-up after bone marrow stromal cell treatment in middle-aged female rats with stroke. Stroke 2007;38(7):2150-6.

STAIR. Recommendations for standards regarding preclinical neuroprotective and restorative drug development. Stroke 1999;30(12):2752-8.

STAIRIII. Recommendations for clinical trial evaluation of acute stroke therapies. Stroke 2001;32(7):1598-606.

Starkey ML, Barritt AW, Yip PK, Davies M, Hamers FP, McMahon SB, Bradbury EJ. Assessing behavioural function following a pyramidotomy lesion of the corticospinal tract in adult mice. Exp Neurol 2005;195(2):524-39.

Tamura A, Kirino T, Sano K, Takagi K, Oka H. Atrophy of the ipsilateral substantia nigra following middle cerebral artery occlusion in the rat. Brain Res 1990;510(1):154-7.

Tracey DJ, Waite ME. Somatosensory System. In: Press A, editor. The rat nervous system 2nd Edition. Sydney: Academic Press; 1995. p 689-700.

van Lookeren Campagne M, Thibodeaux H, van Bruggen N, Cairns B, Gerlai R, Palmer JT, Williams SP, Lowe DG. Evidence for a protective role of metallothionein-1 in focal cerebral ischemia. Proc Natl Acad Sci U S A 1999;96(22):12870-5.

Virley D, Beech JS, Smart SC, Williams SC, Hodges H, Hunter AJ. A temporal MRI assessment of neuropathology after transient middle cerebral artery occlusion in the rat: correlations with behavior. J Cereb Blood Flow Metab 2000;20(3):563-82.

Ward NM, Sharkey J, Brown VJ. Assessment of sensorimotor neglect after occlusion of the middle cerebral artery in the rat. Behav Neurosci 1997;111(5):1133-45.

Zhang L, Li Y, Zhang C, Chopp M, Gosiewska A, Hong KW. Delayed administration of human umbilical tissue-derived cells improved neurological functional recovery in a rodent model of focal ischemia. Stroke 2011;42(5):1437-44. 


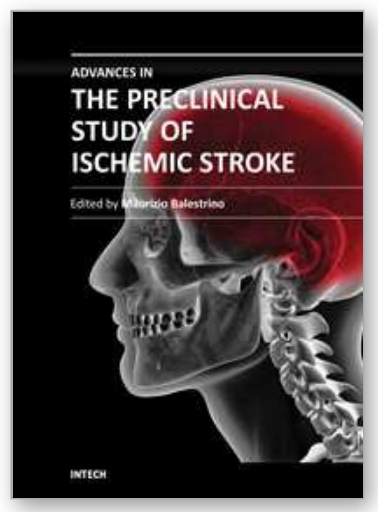

\author{
Advances in the Preclinical Study of Ischemic Stroke \\ Edited by Dr. Maurizio Balestrino
}

ISBN 978-953-51-0290-8

Hard cover, 530 pages

Publisher InTech

Published online 16, March, 2012

Published in print edition March, 2012

This book reports innovations in the preclinical study of stroke, including - novel tools and findings in animal models of stroke, - novel biochemical mechanisms through which ischemic damage may be both generated and limited, - novel pathways to neuroprotection. Although hypothermia has been so far the sole "neuroprotection" treatment that has survived the translation from preclinical to clinical studies, progress in both preclinical studies and in the design of clinical trials will hopefully provide more and better treatments for ischemic stroke. This book aims at providing the preclinical scientist with innovative knowledge and tools to investigate novel mechanisms of, and treatments for, ischemic brain damage.

\title{
How to reference
}

In order to correctly reference this scholarly work, feel free to copy and paste the following:

Valentine Bouet and Thomas Freret (2012). A Master Key to Assess Stroke Consequences Across Species: The Adhesive Removal Test, Advances in the Preclinical Study of Ischemic Stroke, Dr. Maurizio Balestrino (Ed.), ISBN: 978-953-51-0290-8, InTech, Available from: http://www.intechopen.com/books/advances-in-thepreclinical-study-of-ischemic-stroke/a-master-key-to-assess-stroke-consequences-across-species-theadhesive-removal-test-

\section{INTECH}

open science | open minds

\section{InTech Europe}

University Campus STeP Ri

Slavka Krautzeka 83/A

51000 Rijeka, Croatia

Phone: +385 (51) 770447

Fax: +385 (51) 686166

www.intechopen.com

\section{InTech China}

Unit 405, Office Block, Hotel Equatorial Shanghai

No.65, Yan An Road (West), Shanghai, 200040, China

中国上海市延安西路65号上海国际贵都大饭店办公楼405单元

Phone: +86-21-62489820

Fax: $+86-21-62489821$ 
(C) 2012 The Author(s). Licensee IntechOpen. This is an open access article distributed under the terms of the Creative Commons Attribution 3.0 License, which permits unrestricted use, distribution, and reproduction in any medium, provided the original work is properly cited. 